\title{
俄罗斯北部泰米尔半岛褶波带岩床和玄武岩岩石磁学 及磁各向异性
}

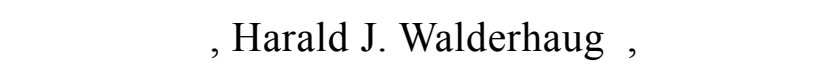

(1) 太原理工大学计算机学院, 太原 030024;

(2) Department of Earth Sciences, University of Bergen, Allègaten 41, 5007 Bergen, Norway;

(3) 中国地质大学(北京)矿产资源与地质过程国家重点实验室, 北京 100083;

(4) 太原理工大学物理系, 太原 030024

E-mail: zhang.shuwei@163.com

2007-08-14 收稿, 2007-11-22 接受

挪威研究基金、EUROPROBE、瑞典北极研究项目基金(SWEDARP)和挪威教育贷款基金(编号: 1921471)资助项目

摘要 对泰米尔半岛东南部 23 个采点的样品进行了磁性测量, 这些样品以明显变形的火 成岩为主. 通过岩石磁学技术和反射光显微镜分析, 发现细粒铁磁铁矿及磁铁矿是岩床 的主要磁组构载体, 而颗粒较大一点的含针低至中等的铷磁铁矿是玄武岩流的主要磁性 矿物. 为了观察研究实验室加热对火成岩磁化率各向异性性质的影响, 对 180 个没有加 热处理过的样品和 128 个在古地磁研究中被热退磁至 $600^{\circ} \mathrm{C}$ 的样品进行了磁化率各向异 性测量, 研究发现实验室加热对这些火成岩样品的各向异性变化影响很大.

关键词

岩床

玄武岩

磁组构

岩石磁学

泰米尔
泰米尔半岛位于欧洲大陆北部边缘，在拉普帖 夫海和喀拉海之间, 对西伯利亚北部的地质研究有 着重要意义. 它包括原生代至白严纪的火成岩、变质 岩、沉积岩(图 1). 对泰米尔半岛火成岩的研究不仅 有利于多学科的发展, 而且有助于理解欧洲北部更 广阔区域范围内的构造演化. 对泰米尔半岛南部火 成岩体 $\left(75^{\circ} \mathrm{N}, 100^{\circ} \mathrm{E}\right.$ )(图 1)进行岩石磁学研究, 既能 够更好地确定磁性载体以及它们对平均磁化率和磁 各向异性的相对贡献, 又为解释稳定剩磁的获得时 间和稳定性提供了证据. 在岩石磁学分析的基础上, 我们可以揭示与初始矿物颗粒大小变化有关的磁性 质、与钛磁铁矿的差异性有关的次生化学蚀变、初始 冷却速率的不同 ${ }^{[1 \sim 4]}$. 在实验室加热泰米尔火成岩是 为了获得具有磁化率主轴取向更好的磁化率各向异 性组构 $[2,3,5]$. 对加热后和加热前的磁组构进行对比, 发现热处理使得磁化率各向异性发生了变化, 这种 变化对所有样品来说并不总是相应于简单的磁组构 的增强. 我们做这项研究的一个主要目的就是更好
地理解加热后的磁性质和磁组构的变化.

\section{1 地质背景和采样}

泰米尔半岛传统上被划分为北、中、南泰米尔 3 个区域(图 1(b)). 泰米尔的火成岩侵入了石炭-早三叠 的老系列, 并以北东东-南西西(NEE-SWW)走向为袹 皱轴与这个系列共同发生了褶坡作用，早侏罗的沉 积系列不整合覆盖于与其共同袑皱的火成岩以及石 炭-早三叠的系列之上, 限定了岩浆作用和袑䏢作用 的时间(图 1). 虽然在泰米尔南部已经发现了晚二叠早三叠的碎屑岩 ${ }^{[8,9]}$, 但在我们研究的区域并没有早 三叠的碎屑沉积岩. 岩床的走向主要是在 $84^{\circ} \sim 119^{\circ}$ 变 化, 倾角小, 一般低于 $28^{\circ}, 3 \sim 5 \mathrm{~m}$ 厚; 玄武岩走向主 要是 $59^{\circ} \sim 80^{\circ}$, 倾角略大, 一般在 $23^{\circ} \sim 36^{\circ}$ 变化, $3 \sim 5 \mathrm{~m}$ 厚, 可以观察到保存好的柱状节理. 泰米尔火山岩的 定位受到了北北西-南南东(NNW-SSE)走向的褔发作 用、东西走向的逆冲断层、东西走向的走滑断层的控 制, 这些断层和䙒坡, 与北泰米尔和中南泰米尔(即北 西伯利亚)发生碰撞而产生的北北西-南南东( NNW- 


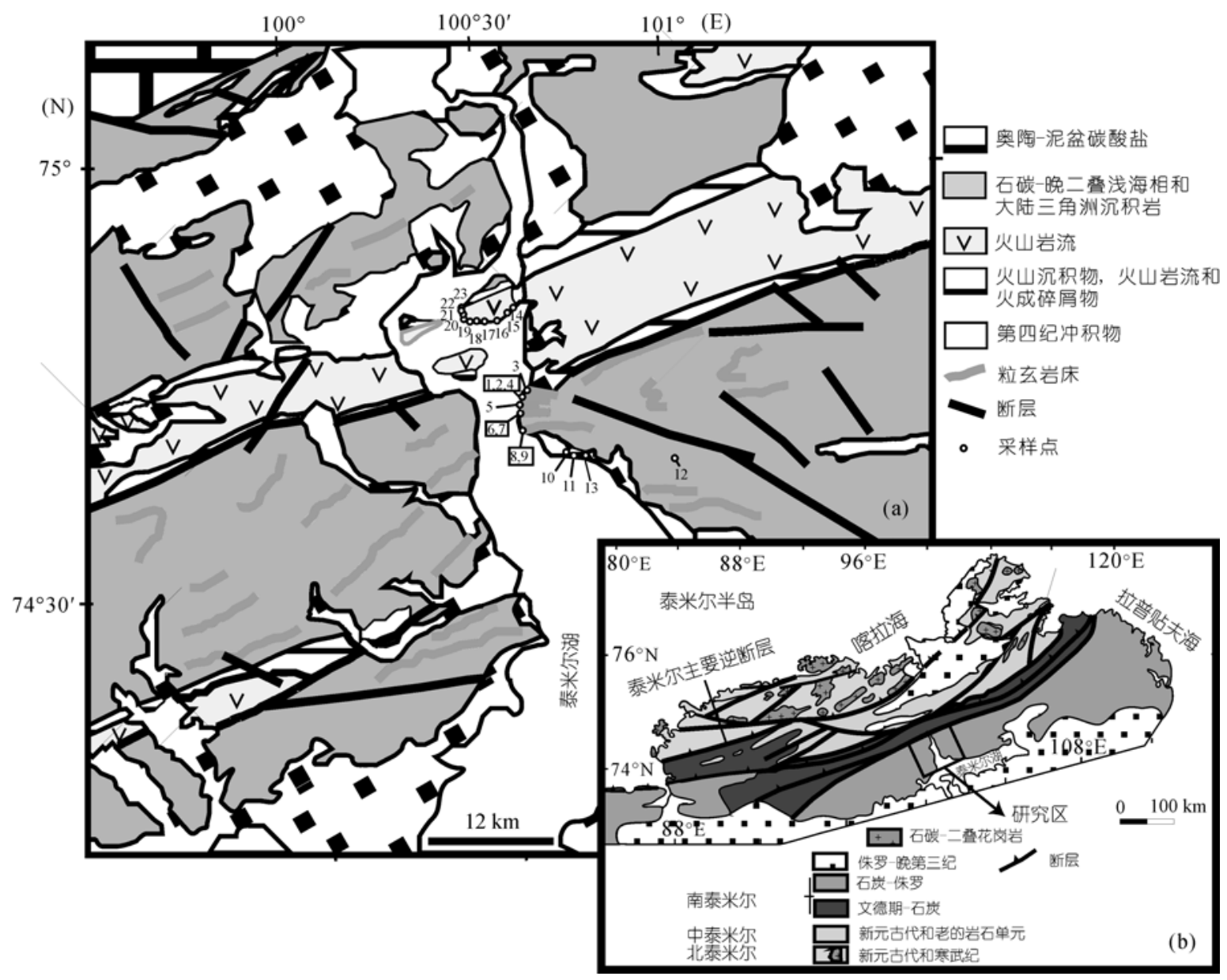

图 1

(a) 研究区域地质图 [6]采样点位置(采样点以 $1,2, \cdots$ 等标注); (b) 泰米尔半岛在北西伯利亚的位置图 [7], 研究区在泰米尔河北部并用矩形标识

SSE)走向的挤压有关 ${ }^{[6,10]}$. 所研究的喷发岩和侵入岩 的年龄通常被认为是二叠-三叠时期 $[6]$; 岩床的古地 磁年龄是 230 220 $\mathrm{Ma}^{[11]}$ ，玄武岩的古地磁年龄是 250 220 $\mathrm{Ma}^{[11]}$.

通过野外打钻和手工取样, 在 23 个采点从圆柱 状钻心共准备了样品 308 块. 打钻时用磁罗盘和太阳 罗盘定向, 并尽可能用太阳罗盘以避免受到被采样 的岩石中剩余磁化强度的磁干扰.

\section{2 磁性矿物学}

由于磁化率各向异性与岩石中的磁性矿物有关, 而这些磁性矿物具有不同的磁性行为, 所以判定岩 石中的磁性矿物非常重要. 在这项研究中, 我们用多 种岩石磁学方法来分析磁性矿物性质, 如: 磁滞曲 线、反射光显微镜和热磁曲线, 利用这些技术可以识 别铁磁矿物种类( 磁铁矿和赤铁矿)、颗粒大小以及它
们对整个岩石磁化率的相对贡献.

\section{1 热磁分析和反射光显微镜方法}

利用居里转换平衡仪对 34 个样品进行热磁分析, 测量磁性矿物的居里温度 $\left(T_{\mathrm{c}}\right)$, 样品在 0.7 Tesla磁场 中被加热至 $700^{\circ} \mathrm{C}$ 然后冷却, 历时 $90 \mathrm{~min}$, 磁化强度 随温度而变化. 对岩床来说, 磁滞曲线几乎可逆, $T_{\mathrm{c}}$ 接近 $580^{\circ} \mathrm{C}$, 说明低钛-磁铁矿甚至磁铁矿是主要载磁 矿物(图 2 中采点 $\mathrm{S} 8$ ). 而玄武岩流相对变化较大, 热磁 曲线不可逆, $T_{\mathrm{c}}$ 在 $435^{\circ} \mathrm{C}$ (图 2 中采点 $\mathrm{S} 14$ )至 $580^{\circ} \mathrm{C}$ (图 2 中采点S17)之间变动, 说明玄武岩中磁性矿物的钛 含量相对较高 $[11,12]$.

利用岩石样品的磨光面在显微镜下鉴定不透明 矿物, 可以帮助确定引起磁化的原因以及影响不透 明矿物相的后期低温氧化作用, 使磨光面浸在油中 可以提高清晰度. 图 3 显示了一些典型的矿物结构, 

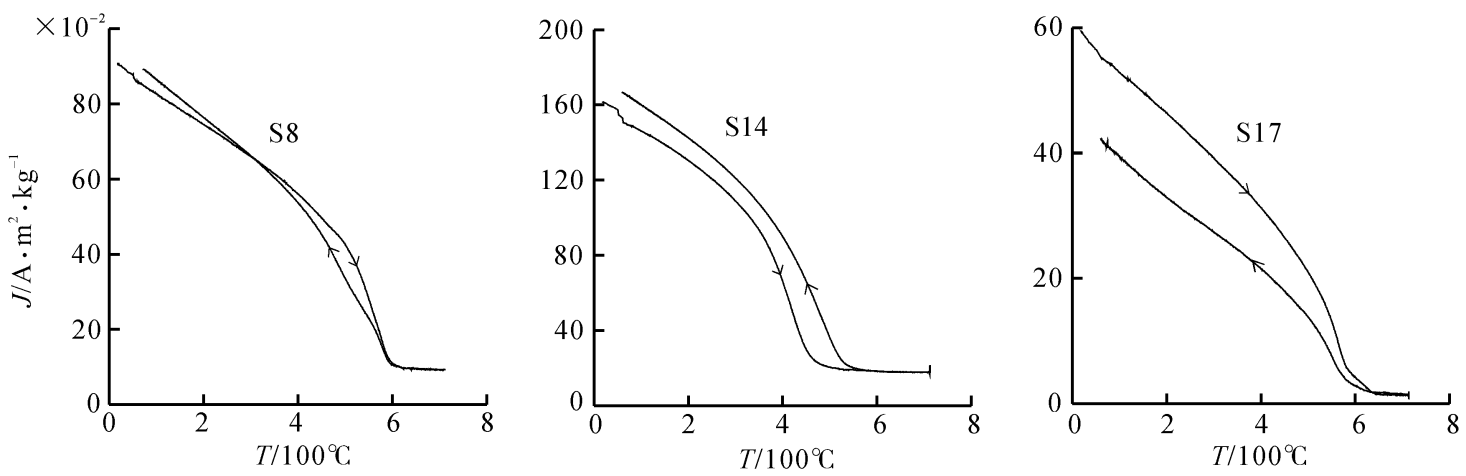

图 2

岩床(采点 $\mathrm{S} 8$ )和玄武岩(采点 $\mathrm{S} 14$ 和 $\mathrm{S} 17$ )的典型的热磁曲线，箭头表示加热或冷却的方向
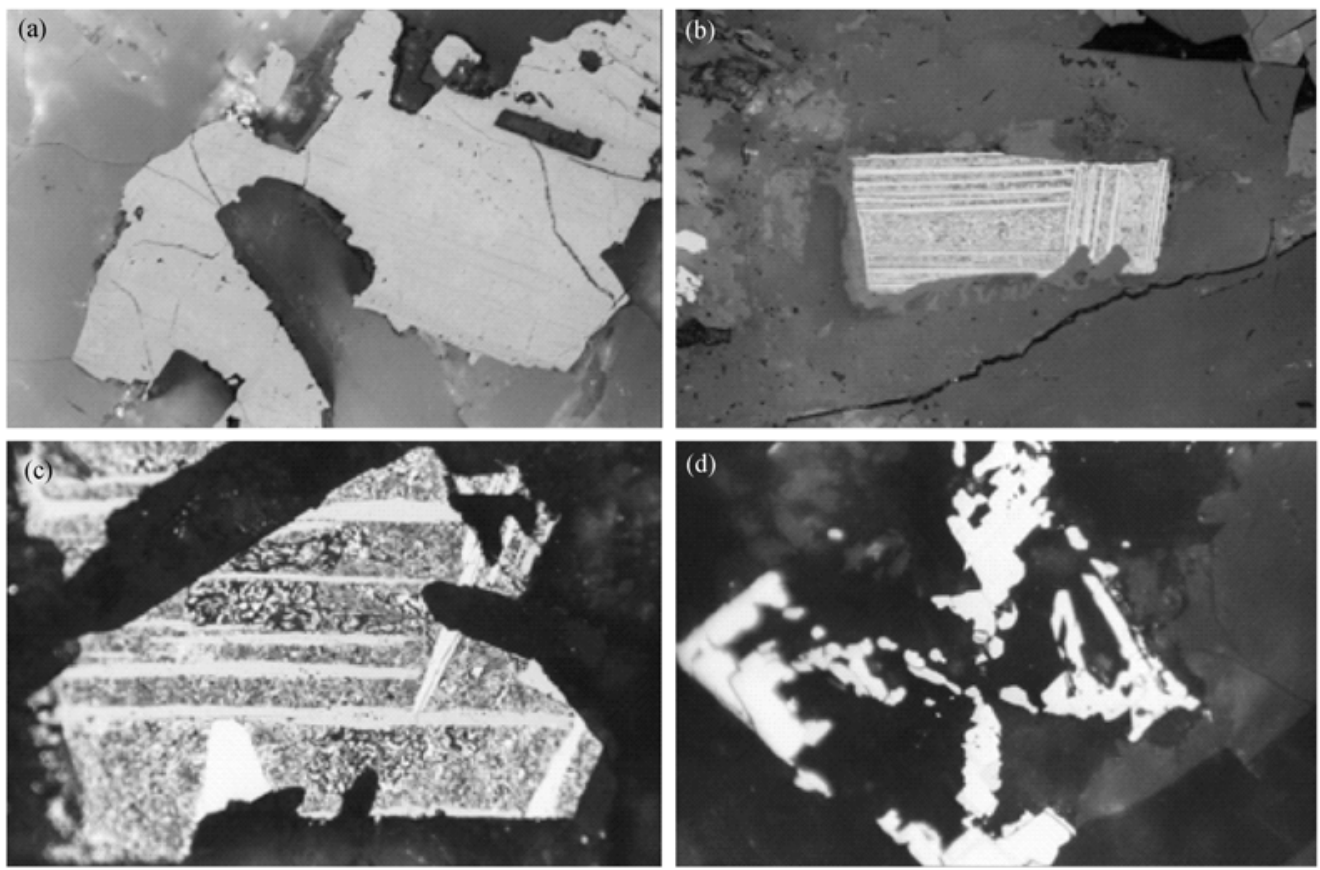

图 3

有代表性的显微镜照片, 显示了典型矿物结构; 岩床(a) (c); 玄武岩(d)

有些图片的拍摄借助了高分辨率数码照相机和油浸 磨光面. 钛磁铁矿是主要磁性矿物. 岩床通常显示含 有相对大的矿物颗粒, 包括钛磁铁矿和有钛铁矿条 带的晶体(三明治结构)(图 3(a) (c)), 这些钛铁矿条带 在偏光显微镜下呈暗褐色，表明初始冷却速度相对 较慢. 图 3(a)显示了一个大的钛磁铁矿晶体，沿着碎 裂处部分蚀变为钛磁赤铁矿; 图 3(b)中的钛铁矿可能 是原生的，也可能是氧化脱溶生成的; 图 3(b), (c)中 钛赤铁矿呈细粒状均匀散布在碎片中。一些收缩裂 隙和细粒状表面指示了低温蚀变作用，这就导致了 磁性矿物颗粒细分成更小的粒子．相反，玄武岩呈现
了均匀的细颗粒的骸晶结构 ${ }^{[11,12]}$ (图 3(d)), 表明了相 对快的冷却速度并且几乎没有发生次生蚀变; 偶尔 会在玄武岩中发现大的钛磁铁矿矿物颗粒。没有观 察到黄铁矿.

\section{2 体积磁性质和磁滞}

岩床中剩磁强度和磁化率(图 4(a), (b))对各采点 平均值分别是 $0.13 \sim 1.33 \mathrm{~A} / \mathrm{m}$ 和 $3.19 \times 10^{-3} \sim 1.44 \times$ $10^{-2} \mathrm{SI}$ (表 1), 玄武岩呈现了较高的剩磁强度和磁化 率, 采点平均值分别是 $0.15 \sim 1.99 \mathrm{~A} / \mathrm{m}$ 和 $5.11 \times 10^{-3} \sim$ $2.02 \times 10^{-2} \mathrm{SI}($ 表 1$)$. 这些数值说明磁化率主要由铁 磁矿物控制 ${ }^{[3]}$, 因而顺磁矿物的贡献基本可以排除, 

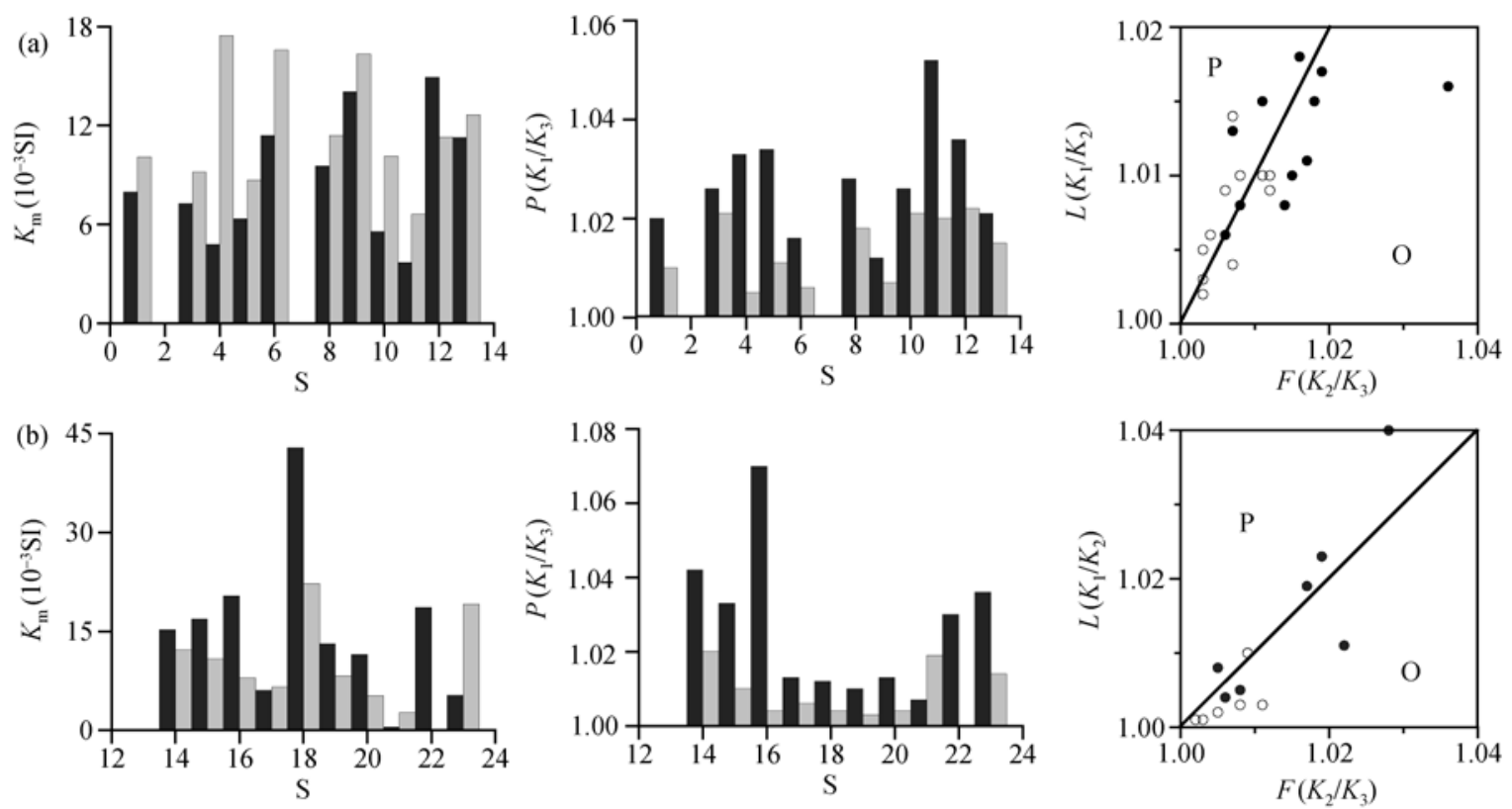

图 4

加热前(黑色)及加热后(灰色或空心圆)岩床(a)和玄武岩(b)的平均磁化率 $\left(K_{\mathrm{m}}\right)$, 磁各向异性度 $(P)$ 和弗林图. 片理 $(F)$ 与线理 $(L)$ 之比表明磁化率椭球各向异性的形状. $\mathrm{S}$ 表示采点, $\mathrm{O}$ 表示扁平椭球体, $\mathrm{P}$ 表示拉长椭球体

表 1 被测量的 $N$ 个样品的磁化率各向异性参数

\begin{tabular}{|c|c|c|c|c|c|}
\hline \multicolumn{6}{|l|}{ 未加热 } \\
\hline 岩石类型 & $N$ & $K_{\mathrm{m}}\left(10^{-3} \mathrm{SI}\right)$ & $P$ & $L$ & $F$ \\
\hline 岩床 & 106 & $8.806 \pm 5.617$ & $1.022 \pm 0.012$ & $1.010 \pm 0.006$ & $1.011 \pm 0.006$ \\
\hline 玄武岩 & 28 & $12.644 \pm 7.537$ & $1.052 \pm 0.019$ & $1.028 \pm 0.013$ & $1.027 \pm 0.010$ \\
\hline \multicolumn{6}{|l|}{ 加热 } \\
\hline 岩石类型 & $N$ & $K_{\mathrm{m}}\left(10^{-3} \mathrm{SI}\right)$ & $P$ & $L$ & $F$ \\
\hline 岩床 & 63 & $11.859 \pm 5.423$ & $1.009 \pm 0.006$ & $1.004 \pm 0.003$ & $1.002 \pm 0.003$ \\
\hline 玄武岩 & 40 & $10.030 \pm 6.951$ & $1.019 \pm 0.004$ & $1.012 \pm 0.003$ & $1.008 \pm 0.002$ \\
\hline
\end{tabular}

因为顺磁矿物的平均磁化率是 $5 \times 10^{-4}$ SI数量级 ${ }^{[13,14]}$, 比绝大多研究的样品磁化率值低 2 个数量级. 热磁分 析(图 2) 显示岩床具有一致的很高的 $T_{\mathrm{c}}$, 说明磁性矿 物有相对一致的化学组成. 绝大多数样品具有较高 的Königsberger 比值 $Q^{\prime}>1$ (图 5(a)), 说明细粒矿物(单 畴或假单畴)是稳定剩磁的携带者, 而一些样品(采点 S3-6, 13 和 14)具有较低比值 $Q^{\prime}<1$ (图 5(a)), 说明较大 的磁性矿物颗粒(较大假单畴或多畴)对剩磁有所贡 献. 通过磁滞测量可以推断颗粒大小和矿物学性质 的变化, 我们用振动样品磁力仪(Molspin VSM)对不 同采点的样品做磁滞实验, 这个仪器足以使钛磁铁 矿和钛磁赤铁矿达到饱和, 但由于其最大磁场是 1.14 $\mathrm{T}^{[3]}$, 所以不足够使细粒赤铁矿饱和. 表 2 中有 2 个比
值 $J_{\mathrm{rs}} / J_{\mathrm{s}}$ 和 $H_{\mathrm{cr}} / H_{\mathrm{c}}$, 其中 $J_{\mathrm{rs}} / J_{\mathrm{s}}$ 是饱和剩磁磁化强度与饱 和磁化强度之比, $H_{\mathrm{cr}} / H_{\mathrm{c}}$ 是剩磁矫顽力与矫顽力之比, 图 5(b) (d)显示了典型的几条磁滞曲线. $J_{\mathrm{rs}} / J_{\mathrm{s}}$ 和 $H_{\mathrm{cr}} / H_{\mathrm{c}}$ 以及 $H_{\mathrm{c}}$ 表明大多数样品包含假单畴磁性矿物 ${ }^{[15]}$. 然 而，这两种火成岩可能含有不同颗粒大小的多种磁 性矿物 ${ }^{[15]}$, 并且根据Dunlop ${ }^{[16,17]}$ 最近的修订标准认 为Day图中假单畴 ${ }^{[15]}$ 是单畴和多畴的混合体，所以 不能排除这些磁性矿物是单畴和多畴的混合. 如表 2 所示, 岩床的 $J_{\mathrm{rs}} / J_{\mathrm{s}}$ 和 $H_{\mathrm{cr}} / H_{\mathrm{c}}$ 分别是 0.2 0.3 和 1.5 2.5, 矫顽力 $H_{\mathrm{c}} 13.5 \sim 20 \mathrm{mT}$, 大多数玄武岩的 $J_{\mathrm{rs}} / J_{\mathrm{s}}, H_{\mathrm{cr}} / H_{\mathrm{c}}$ 和 $H_{\mathrm{c}}$ 分别是 $0.1 \sim 0.3,1.6 \sim 2.6$ 和 5.4 18.2 $\mathrm{mT}$. 磁性矿物 颗粒变细很可能导致岩床比玄武岩样品具有更高的 $H_{\mathrm{c}}$ 和 $J_{\mathrm{r} \mathrm{s}} / J_{\mathrm{s}}$ 值, 这说明岩床含有 

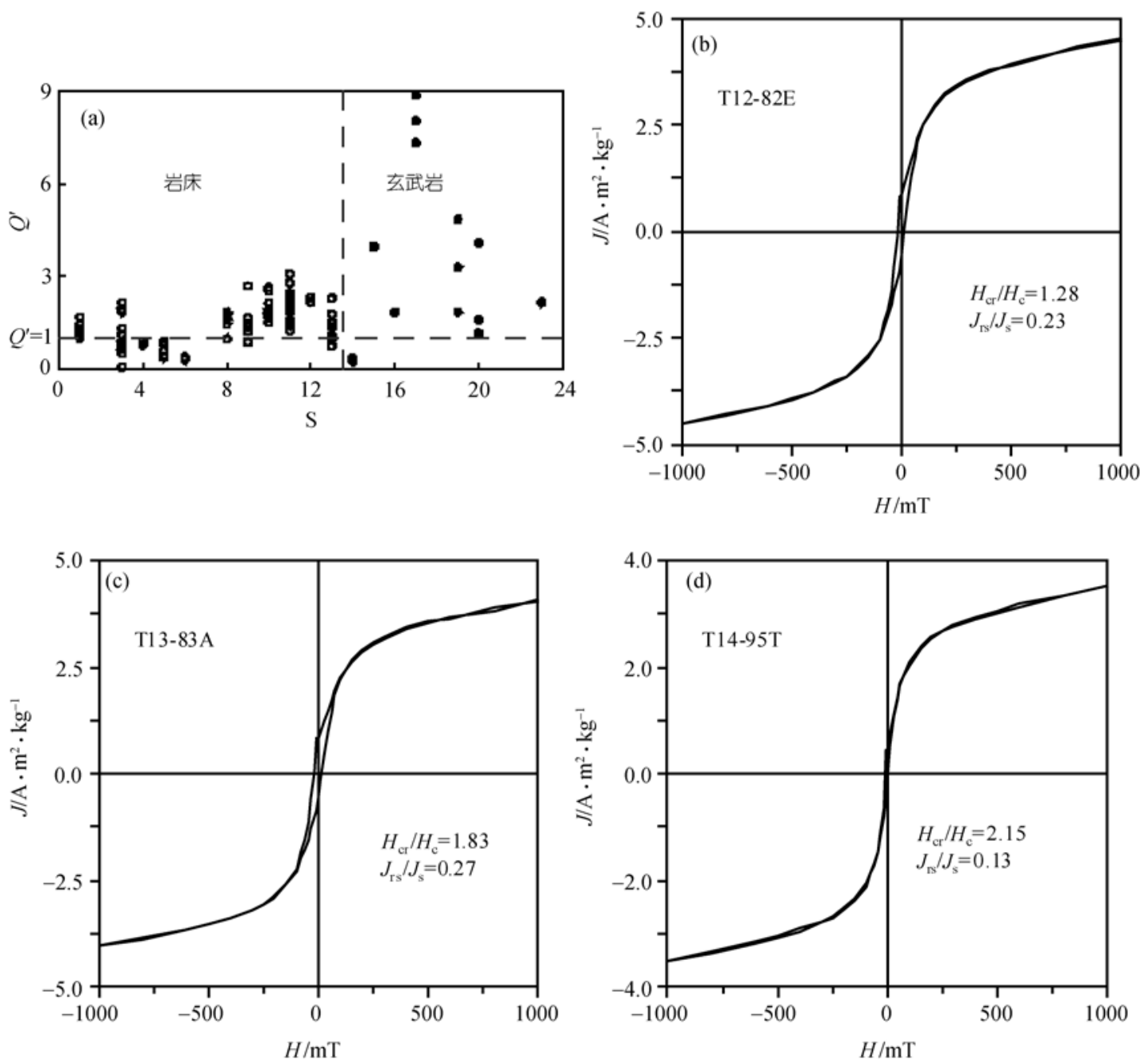

图 5

(a) $Q^{\prime}$ 值图说明岩床和玄武岩的磁畴, $Q^{\prime}=\left(10^{7} / 4 \pi\right) \times(\mathrm{Jr} / \mathrm{kH}) \mathrm{SI}$, 其中 $\mathrm{H}$ 是地球磁场的大小, $\mathrm{S}$ 表示采点; (b)和(c) 代表了岩床的磁滞曲线;

(d) 代表玄武岩磁滞曲线; 样品 T12-82E, T13-83A 和 T14-95 T 的矫顽力分别是 $H_{\mathrm{c}}=15.04,17.25$ 和 $7.35 \mathrm{mT}$

表 2 岩床和玄武岩的磁滞参数

\begin{tabular}{ccccc}
\hline 岩石类型 & $\mathrm{N}$ & $H_{\mathrm{c}} / \mathrm{mT}$ & $J_{\mathrm{rs}} / J_{\mathrm{s}}$ & $H_{\mathrm{cr}} / H_{\mathrm{c}}$ \\
\hline 岩床 & 22 & $16.45 \pm 3.08$ & $0.32 \pm 0.19$ & $1.22 \pm 0.96$ \\
玄武岩 & 17 & $11.81 \pm 6.37$ & $0.21 \pm 0.11$ & $2.02 \pm 1.93,9.25,13.87$ \\
\hline
\end{tabular}

较小的假单畴, 或单畴和多畴的混合物, 玄武岩含较 大的假单畴磁性矿物. 而岩床仍然可能含有假单畴和 多畴的混合物 ${ }^{1)}$. 玄武岩中有两个样品 $H_{\mathrm{cr}} / H_{\mathrm{c}}$ 值较高, 说明超顺磁铁矿有可能存在 ${ }^{[15 ~ 17]}$.

总之, 岩床比玄武岩表现了更均一的磁性质, 较 小的假单畴, 或单畴和多畴混合的磁性矿物 (钛磁铁
矿或磁铁矿)是主要剩磁载体, 钛铁矿条带相应于缓 慢冷却. 玄武岩普遍含钛磁铁矿, 有时可见磁铁矿, 细的骸晶结构的矿物颗粒暗示了快速冷却.

\section{3 岩床和玄武岩的磁化率各向异性}

不同的矿物和磁畴对样品总的各向异性都有贡 献, 因此磁化率各向异性是一个复杂的现象. 在卑尔 根大学古地磁实验室, 用 KLY-2 卡帕桥对 180 个没有 加热的样品和 128 个逐步加热至 $600^{\circ} \mathrm{C}$ 的样品进行磁 化率各向异性测量. 分别用磁化率各向异性参数 $K_{1}$, $K_{2}$ 和 $K_{3}$ 来定义最大、中间和最小磁化率主轴.

1) Zhang S W, Walderhaug H J, Yang Y J. Magnetic fabric and its significance in igneous rocks from Taimyr fold-belt, Arctic Siberia. Geophys J Int, 待发表 
如图 4(a)和表 1 所示, 由于化学成分转化、新氧 化物生成、单畴-假单畴-多畴之间的转化引起了矿物 颗粒大小变化 ${ }^{[3,4,18 ~ 20]}$, 所以在加热至 $600^{\circ} \mathrm{C}$ 以上时 岩床磁化率增高; 在原始晶体内新生成的铁磁性矿 物会导致磁化率强烈增加 ${ }^{[3,4,18 ~ 20]}$, 这些铁磁性矿物 是由非磁性矿物或弱磁性矿物转化而来. 相反, 玄武 岩磁化率降低, 说明铁磁性矿物由于加热时发生蚀 变、矿物间转化以及单畴-假单畴的出现 ${ }^{[3,4,16,17,19]}$, 而 转变成为非磁性矿物或弱磁性矿物(例如磁铁矿氧化 成赤铁矿). 晶体缺陷的均匀分布以及空缺处新的阳 离子填充引起的转化可使磁化率发生很大改变 ${ }^{[20]}$. 正 如具有原生磁组构的火成岩样品那样, 两种岩石的磁 化率各向异性 $\left(P=K_{1} / K_{3}\right)$ 通常很低(图 4(a), (b); 表 1), 大多数岩床样品的 $P$ 在加热前为 $1.010 \sim 1.034$, 在加热 后为 $1.003 \sim 1.015$, 而大多玄武岩样品加热前和加热后 $P$ 值分别为 $1.033 \sim 1.071$ 和 $1.015 \sim 1.023^{[14,21,22]}$. 加热引 起了 $P$ 的普遍降低(图 4(a), (b); 表 1), 可能由于去掉 了构造作用和低温蚀变导致的次生各向异性 ${ }^{[23]}$, 同 时暗示了被次生磁化率各向异性成分掩盖的原生定 位的影响 ${ }^{[3]}$, 此外, 磁性矿物蚀变和新矿物的出现会 进一步降低 $P$. 加热后线理度 $\left(L=K_{1} / K_{2}\right)$ 和片理度 $(F=$ $\left.K_{2} / K_{3}\right)$ 下降, 在岩床和未加热的玄武岩中, 扁平的和 拉长的磁化率各向异性椭球数量大体相等, 即磁片 理并不比磁线理占优势, 这与Knight和Walker ${ }^{[24]}$ 在夏 威夷基性岩墙中发现的结果相似, 但加热的玄武岩 的磁组构以扁平的磁化率各向异性椭球形状为主(图 4(b); 图 6). 在岩床和未加热的玄武岩中某些采点显 示扁平磁化率各向异性椭球, 某些显示拉长的椭球 形状, 还有一些显示三轴椭球类型(采点 $\mathrm{S} 11$ 和未加 热的采点 $\mathrm{S} 17,19$ ).

图 6 显示了磁化率各向异性主轴的分布, 看起来 受热时岩床采点 $\mathrm{S} 1, \mathrm{~S} 5, \mathrm{~S} 10, \mathrm{~S} 11$ 和 $\mathrm{S} 13$ 中倾角较小的 $K_{1}$ 轴从平行层面向垂直于层面方向移动, $K_{2}$ 和 $K_{3}$ 轴 向平行层面方向挪动, 因此加热至 $600^{\circ} \mathrm{C}$ 得到了增强 的 $K_{1}$ 轴取向; 从而椭球形状也发生改变, 如图 4(a), (b) 和图 6 所示, 加热前采点 $\mathrm{S} 1$ 和 $\mathrm{S} 5$ 主要显示拉长椭球 形状, 采点 $\mathrm{S} 10, \mathrm{~S} 11$ 和 $\mathrm{S} 13$ 显示了扁平椭球, 加热后 采点 $\mathrm{S} 10$ 和 $\mathrm{S} 13$ 显示拉长椭球, 其他采点的拉长椭球 和扁平椭球数量基本相等; 其中, 采点 $\mathrm{S} 5$ 中 $K_{1}$ 和 $K_{2}$ 轴通过互换位置而发生 $90^{\circ}$ 移动, 而 $K_{3}$ 轴基本稳定;
当矿物颗粒由单轴形状的磁化率各向异性控制时 ${ }^{[3,25]}$, 假单畴或大一点的矿物颗粒向较小的颗粒 (单畴)转 换能够引起 $K_{1}$ 和 $K_{3}$ 轴互换位置, 这种机制能够解释 椭球形状和 $K_{3}$ 轴取向的变化, 与我们的观察一致, 即 岩床含有假单畴矿物或单畴和多畴矿物混合物 ${ }^{1)}$. 玄 武岩采点 $\mathrm{S} 17$ 和 $\mathrm{S} 19$ 的磁化率主轴也发生了明显移动, 其 $K_{1}$ 和 $K_{2}$ 轴互换位置, 有趣的是, $K_{3}$ 轴相对保持稳定, 受热后的磁组构较加热前的差一些; 采点S16 在受热 前显示拉长椭球, 受热后获得了增强的磁组构并且 扁平椭球占优势. 对泰米尔火成岩加热提供了更多 的有关有效磁性质变化的信息, 加热后的磁组构与 初始磁组构有的相似(采点S11), 有的相反 ${ }^{[3,5,18,25]}$, 有的产生了磁化率轴的不同取向 (采点 S5, S13 等). 热处理不仅可以增强加热前的磁组构, 而且也可以 使加热前的初始磁组构变得更差; 磁化率主轴和椭 球形状的变化与加热过程中消失或产生的铁磁矿物 相对应 $[18,20]$.

前人已经观察研究了倒置磁组构, 并且认为与

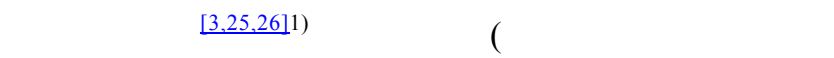
解石 $\left.{ }^{[25,27]}\right)$ 有关. 众所周知, 单畴磁铁矿具有倒置磁 组构 ${ }^{[25]}$, 其 $K_{1}$ 轴垂直于基面(图 4 中采点 $\mathrm{S} 10, \mathrm{~S} 11$ 和 加热的采点 $\mathrm{S} 5$ 等), 恰好相反的是, 正常磁组构的 $K_{3}$ 轴垂直于基面并平行于层面的极(图 6 中未加热采点 S17 和 19). 单畴与假单畴或多畴的混合贡献会产生 中间磁组构, 也就是说, $K_{2}$ 轴垂直于层面, $K_{1}$ 和 $K_{3}$ 轴 在层面上或没有一致的取向 (图 4 中未加热采点 $\mathrm{S} 1$, S5 和S13 等). 在我们的研究中, 通过磁性矿物学已 确定了单畴磁行为是可能存在的, 在磁化率各向异 性测量中它总是会导致磁化率轴的倒置 $[3,18,25,28]$.

\section{4 讨论}

用综合的岩石磁学技术对泰米尔火成岩进行分 析, 发现岩床含有低钛-钛磁铁矿, 玄武岩中主要铁 磁矿物是含钛低至中等的钛磁铁矿及少量赤铁矿. 在埋藏很深的海洋玄武岩 ${ }^{[29]}$ 和浅水喷出岩 ${ }^{[23,30]}$ 中已 发现了低钛-钛磁铁矿. 泰米尔火成岩磁性质变化, 不仅与矿物颗粒大小变动有关, 而且与磁性矿物聚 集程度有关. 在岩床中已经发生了中等(图 3(a))至强 烈(图 3(b), (c))的次生的低温蚀变, 这将使得岩床中 有效的磁性颗粒粒度明显降低, 那么稳定的剩磁携

1) 同第 233 页脚注 


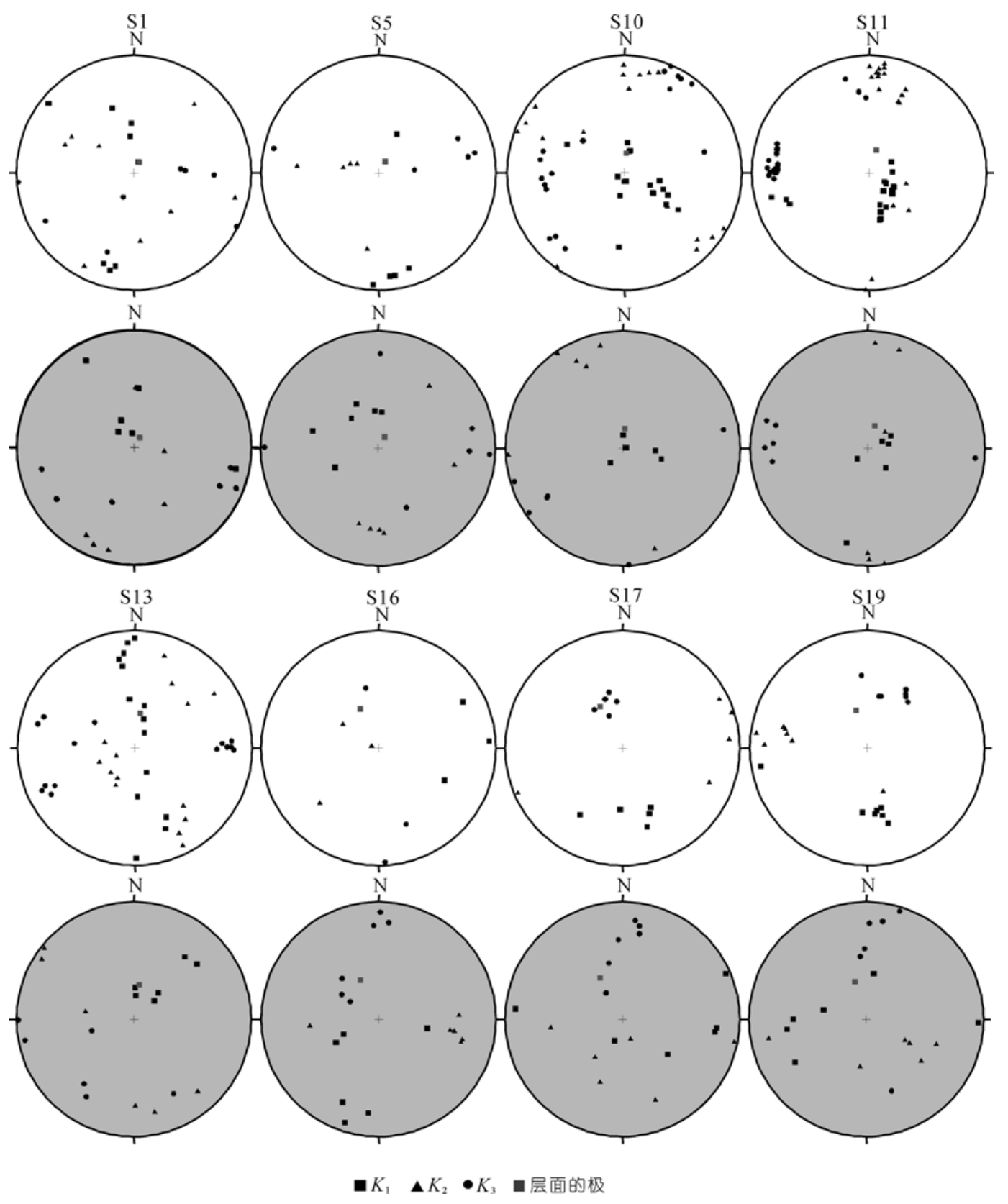

图 6

磁化率各向异性主轴在加热前(空心圆)和加热至 $600^{\circ} \mathrm{C}$ 后(灰圆圈)的投影图, 包括采点 $\mathrm{S} 1, \mathrm{~S} 5, \mathrm{~S} 10, \mathrm{~S} 11, \mathrm{~S} 13, \mathrm{~S} 16, \mathrm{~S} 17$ 和 $\mathrm{S} 19$

带者钛磁铁矿的磁畴就应该是假单畴或单畴. 如果 颗粒较细，容易得到水并且能与水相混合，再加上裂 隙中氧气的作用, 就有助于岩石中氧化作用的进行 [3,18]. 通过光学显微镜观察, 认为岩床中有一些稍微 有点儿次生的磁铁矿 $\left(T_{\mathrm{c}}\right.$ 接近 $\left.580^{\circ} \mathrm{C}\right)$ 在实验室加热前 已经形成. 小颗粒的原生钛磁铁矿不可能是唯一的
磁组构携带者, 同一块岩石样品可能有多种磁性矿 物颗粒共存 ${ }^{1)}$. 即使在岩床中显微镜下可见的磁性矿 物颗粒似乎比玄武岩的大, 但岩床含有单畴和小的 假单畴矿物颗粒 ${ }^{1)}$, 而玄武岩中有效的磁性颗粒可能 更趋向大的假单畴.

铁磁矿物对二叠-三叠的岩床和玄武岩的磁化率

1) 同第 233 页脚注 
各向异性做出了主要贡献. 加热过程中新形成的铁磁 性颗粒或者取代了前期的矿物或者相应于前期矿物的 脱溶产物 $[3,5,18,20,21]$. 加热的泰米尔火成岩的 $P$ 相对降 低, 这与Henry ${ }^{[20]}$ 的结果相反, 但与Walderhaug ${ }^{[3]}$ 的结 果一致. 已有文章研究报道了加热后增强的磁组构 [3,18,25,27], 但加热引起的变化远不是简单地增强或改

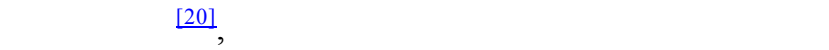
的磁组构产生, 所以加热后的磁组构是一个复合的 磁组构, 与初始磁组构和新产生的磁组构有关 ${ }^{[20,31]}$. 既然岩石是一个复杂的多矿物系统, 磁性质的变化 不能简单解释成一个单一矿物相的解体，而应该考 虑所有矿物相.

目前, 磁组构的解释并不是非常明确 ${ }^{[5]}$. 每个磁 化率各向异性的成分与岩浆定位时的岩浆流动方向、 冷却应力、构造应力有关 ${ }^{[3,28]}$, 这使得任何去得到期 望的初始磁组构的方向的预言都带有不确定性. Ell$\operatorname{wood}^{[31]}$ 认为一些次生的磁组构 $\left(K_{3}\right.$ 轴水平)可能与热 应力有关. 利用计算机模型Dawson和Hargrave ${ }^{[32]}$ 发 现流动的岩墙的 $K_{1}$ 轴指向流向, 而 $K_{3}$ 轴垂直于岩墙 壁, 在静止的岩墙中, $K_{1}$ 轴垂直于岩墙壁. 岩床作为 平坦的侵入构造, 它平行于围岩的层面, 所以岩床的 流动方向不可能垂直于层面, 然而我们所研究的岩 床与第二种情况类似, 即 $K_{1}$ 轴垂直于围岩层面. $K_{2}$ 和 $K_{3}$ 轴方向明显分散, 可以被认为是磁片理度很低的、 磁化率轴取向差的组构. 因此按照以上模型解释岩 床的各向异性是有疑问的, 但所研究的岩床磁组构
可能是由岩浆流控制的原生磁组构. 仍然需要做进 一步的研究来判定磁性矿物的磁畴以便更有效地研 究岩浆的定位.

\section{5 结论}

泰米尔火成岩的平均磁化率 $\left(K_{\mathrm{m}}\right)$ 普遍较高, 岩床 的 $K_{\mathrm{m}}>3.7 \times 10^{-3} \mathrm{SI}$, 玄武岩的 $K_{\mathrm{m}}>5.3 \times 10^{-3} \mathrm{SI}$ (表 1 和 图 4). 这些岩石中磁性质的变化是由钛磁铁矿的聚 集程度以及矿物颗粒大小不同引起的. 小颗粒的磁 性矿物是稳定剩磁的携带者.

磁化率各向异性主要由铁磁矿物携带. 细粒(小 一点的假单畴或单畴)和粗粒 (大一点的假单畴或多 畴) 的钛磁铁矿直至纯磁铁矿是岩床的主要磁性矿 物, 然而玄武岩中磁性矿物可能是更趋向于大一点 的假单畴、多畴, 并含钛低至中等的钛磁铁矿. 研究 的岩床中倒置的磁组构(图 6)很可能由单畴的磁性矿 物导致.

加热使磁化率各向异性发生变化, 对所有样品 来说, 这种变化不总是相应于简单的磁组构的增强, 有时候会得到相反结果, 因为加热会导致磁性矿物 的转化、新矿物的产生、新矿物颗粒的出现. 加热后 的磁组构与加热前的磁组构和加热过程中新产生的 矿物引起的磁组构有关. 加热也导致了相应的磁性 参数的变化. 对于加热处理的样品, 应该仔细研究每 个采点在加热后所得到的磁组构数据, 来判定加热 处理是否明显增强了磁组构.

致谢 作者对两位匿名审稿人提出的宝贵意见和建议, 以及在实验过程中卑尔根大学古地磁实验室的所有工作人员给 予的帮助，张世红教授、刘青松研究员和 Yu Yongjae 博士给予的建议，韩以贵博士等给予的帮助，在此一并致 谢.

\section{参考文献}

1 Petersen N. Notes on the variation of magnetization within basalt lava flows and dikes. Pageophys, 1976, 114: 177-193 [doi]

2 Ellwood B B, Balsam W, Burkart B, et al. Anomalous magnetic properties in rocks containing the mineral siderite: Paleomagnetic implications. J Geophys Res, 1986, 91: 12779-12790

3 Walderhaug H J. Rock magnetic and magnetic fabric variations across three thin alkaline dikes from Suunhord-land, Western Norway; influence of initial mineralogy and secondary chemical alterations. Geophys J Int, 1993, 115: 97-108 $\underline{\text { [doi] }}$

4 Liu Q S, Banerjee K S, Jackson J M, et al. New insights into partial oxidation model of magnetites and thermal alteration of magnetic mineralogy of the Chinese loess in air. Geophys J Int, 2004, 158: 506-514 [doi]

5 Tarling D H, Hrouda F. The Magnetic Anisotropy of Rocks. London: Chapman \& Hall, 1993

6 Bezzubtsev V V, Malitch N S, Markov F G, et al. Geological map of mountainous Taimyr, 1:500000. Ministry of Geology of the USSR. Ministry of Geology of the Russian Federation (RFSFR), Krasnoyarskgeologia, Krasnoyarsk (in Russian), 1983 
7 Torsvik T H, Andersen T B. The Taimyr fold belt, Arctic Siberia: Timing of prefold remagnetisation and regional tectonics. Tectonophysics, 2002, 352: 335-348 [doi]

Egorov A Yu, Kulikova L I. Stratigraphic position of the Early. Triassic traps on the Taimyr Peninsula. Trudy IgiG SO AN SSSR (in Russian), 1989, 732: 91-101

9 Mogucheva N K, Betekhtina O A. Urgent problems of the stratigraphy of the continental Siberian Triassic. Geol Geofiz, 1998, 39: $293-302$

10 Inger S, Scott R A, Golionko B G. Tectonic evolution of the Taimyr Peninsula, northern Russia: Implications for Arctic continental assembly. J Geol Soc, 1999, 156: 1069-1072[doi]

11 Walderhaug H J, Eide E A, Scott R A, et al. Palaeomagnetism and ${ }^{40} \mathrm{Ar} /{ }^{39} \mathrm{Ar}$ geochronology from the South Taimyr igneous complex, Arctic Russia: A Middle-Late Triassic magmatic pulse after Siberian flood-basalt volcanism. Geophys J Int, 2005, 163: 1-17 张世红, 李鹏举, 王立波. 吉林省吉舒玄武岩及其围岩磁组构研究. 地球物理学报, 1994, 37(增刊 I): 316一 323

Wagner J J, Hedley F G, Steen D, et al. Magnetic anisotropy and fabric of some progressively deformed ophiolitic grabbros. J Geophys Res, 1981, 86: 307-315

Hrouda F. Magnetic anisotropy of rocks and its application in geology and geophysics. Surv Geophys, 1982, 5: 37—82 [doi]

Day R, Fuller M, Schmidt V A. Hysteresis properties of titanomagnetites: grain size and compositional dependence. Phys Earth Planet Inter, 1977, 13: 260-267[ [doi]

16 Dunlop D J. Theory and application of the Day plot $\left(M_{\mathrm{rs}} / M_{\mathrm{s}}\right.$ versus $\left.H_{\mathrm{cr}} / H_{\mathrm{c}}\right): 1$. Theorical curves and tests using titanomagnetite data. J Geophys Res, 2002, 107: 10.1029/2001 JB000487

17 Dunlop D J. Theory and application of the Day plot $\left(M_{\mathrm{rs}} / M_{\mathrm{s}}\right.$ versus $\left.H_{\mathrm{cr}} / H_{\mathrm{c}}\right) 2$. Application to data for rocks, sediments, and soils. J Geophys Res, 2002, 107: 10.1029/2001 JB000487

Pan Y X, Zhu R X, Banerjee S K, et al. Rock magnetic properties related to thermal treatment of siderite: Behavior and interpretation. J Geophys Res, 2000, 105: 783-794[doi]

Hirt A M, Gerhring A U. Thermal alteration of the magnetic mineralogy in ferruginous rocks. J Geophys Res, 1991, 96: 9947—9953 Henry B, Jordanova D, Jordanova N, et al. Anisotropy of magnetic susceptibility of heated rocks. Tectonophysics, 2003, 366: 241258 [doi]

21 Staudigel H G, Gee G, Tauxe L, et al. Shallow intrusive direction of sheeted dykes in the Troodos ophiolite: Anisotropy of magnetic susceptibility and structural data. Geology, 1992, 20: 841-844 [doi]

22 Callot J P, Geoffroy L, Aubourg C, et al. Magma flow directions of shallow dykes from the East Greenland volcanic margin inferred from magnetic fabric studies. Tectonophysics, 2001, 335: 313-329 [doi]

23 Park J K, Tanczyk E, Debarats A. Magnetic fabric and its significance in the 1400 Ma Mealy diabase dykes of Labrador, Canada. J Geophys Res, 1988, 93: 13689-13704

Knight M D, Walker G P L. Magma flow directions in flows of the Koolau Complex, Oahu, determined from magnetic fabric studies. J Geophys Res, 1988, 93: 4308-4319

Potter D K, Stephenson A. Single-domain particles in rocks and magnetic fabric analysis. Geophys Res Lett, 1988, 15: 1097-1100

Rochette M J, Aubourg C. Rock magnetism and the interpretation of anisotropy of magnetic susceptibility. Rev Geophys, 1992, 30(3): 209-226

Rochette P. Inverse magnetic fabric in carbonate-bearing rocks. Earth Planet Sci Lett, 1988, 90: 229-237 [doi]

Dragoni M, Lanza R, Tallarico A. Magnetic anisotropy produced by magma flow: theoretical model and experimental data from Ferrar dolerite sills (Antarctica). Geophys J Int, 1997, 128: 230-240 [doi]

Smith G M, Banerjee S K. Magnetic properties of basalts from Deep Sea Drilling Project Leg 83: the origin of remanence and its relation to tectonic and chemical evolution. Init Rep DSDP, 1985, 83(b): 347—357

Cockerham R S, Hall J M. Magnetic properties and paleomagnetism of some Leg 33 basalts and sediments and their tectonic implications. J Geophys Res, 1976, 81: 4207-4222

31 Ellwood B B. Anisotropy and magnetic susceptibilty variations in Icelandic columnar basalts. Earth Planet Sci Lett, 1979, 42: 209212[doi]

32 Dawson E M, Hargraves R B. Anisotropy of magnetic susceptibility as an indicator of magma flow directions in diabase dykes (abstract). Eos Trans AGU, 1985, 66: 251 